\title{
Research on Intelligent General Acquisition Controller of Rice Seedling-nursery House in Cold Region Based on MSP430F5438
}

\author{
Tian Fangming, Yi Shujuan, Tan Feng, \\ Wang Xiaoliang, Yan Li, Liu Yingnan \\ College of Information and Technology, Heilongjiang Bayi \\ Agricultural University, Daqing 163319, China \\ mingyu_nd@163.com, 15045975008
}

\author{
Han Donglai \\ Heilongjiang 859 Farm, Jamusi 156326, China
}

\begin{abstract}
In view of the existence of insufficiency of environmental parameter acquisition control system for the current cold rice seedling house, through researching, develop a sampling control system with low power consumption, high precision, strong versatility, to which MSP430F5438 chip processor with low power consumption is the core. Corresponding with the dynamic range of analog pretreatment system, for the construction of hardware platform, use cold rice seedling period environmental parameters and wave characteristics, the different needs of various stages in seedling period, to achieve real-time acquisition of the various environmental parameters and intelligent automatic control of ventilation equipment and irrigation facilities, meet the demands of practical application, the system has a good development prospect based on its high reliability and versatility.
\end{abstract}

Keywords-rice seedling-nursery house; MSP430F5438; acquisition controller; general; high precision

\section{INTRODUCTION}

The commissariat yield in Heilongjiang reclamation area directly relates to food security of China. In Heilongjiang reclamation area, rice planting area is the most extensive, therefore, improve the quality of rice and the security of high and stable yield of rice is very important, and the quality and yield of rice is closely related to other agronomic operation, as the saying goes 'well begun is half done', cultivating strong seedling is the priority among priorities. Seedling rising is a process from seed germination with water culture to seedling, and closely related to environmental conditions, so create a suitable environment for seed germination and seedling growth in the seedling raising process is the most important. Heilongjiang province is located in cold region, annual seedling in April and May, at this time the weather is the most changeful, seedling prone to uneven seedling, bad seedling and seedling death, it is difficult to control the seedling growth with poor uniformity, and the seedling rate is low. The seedling period is divided into the preparations before seeding period, seminal root growth period, first leaves completely long stretch period, transplanting preparation period. The temperature, humidity and other environmental parameters of the requirements in each

echnology plan projects of Heilongjiang agricultural reclamation administration (HNK10A-07-01-05);science and technology research project of Heilongjiang provincial (emphasis) (12511z021) period are very strict. After years of exploration and discovery, only in accordance with the standards and strictly control of environment in Seedling house for raising seedling can famers cultivate strong seedlings. Currently, there is some monitoring equipment on the seedling house, but most have many problems of low precision, bad portability, high cost, therefore, it is urgent to needs to develop a intelligent seedling house control system with high precision, low cost, high reliability, strong universality, in order to ensure the smooth implementation for factory seedling-nursery ${ }^{[1]}$.

\section{SYSTEM FUNCTION DEMAND AND DESIGN THOUGHT}

Through in-depth research and discussion analysis with farm science and technology personnel, intelligent control device of cold rice seedling-nursery house has the following requirements:

It can collect $4 \sim 20 \mathrm{~mA}$ current signal, voltage signal, digital signal, frequency signal, in order to meet the needs of different types of sensor for signal acquisition;

It can record the historical data of air temperature and humidity, soil temperature, soil moisture, light intensity, concentration of $\mathrm{CO} 2$ seedling-nursery house environment parameters with characteristics of real-time, average monthly, daily average ,daily and monthly extreme value, increase data storage capacity to $1 \mathrm{MB}$, rich data recording information can meet different requirements of seedling-nursery house in control field;

Acquisition of environment parameters information and the open state of ventilation and irrigation equipment can be real-time displayed and can query the historical data;

Configuration of threshold value of air temperature and humidity, soil temperature, soil moisture for seedling stage in different stages, when the environmental parameters is lower or higher than the threshold value, the means such as short message by the sound and light used to alarm, and can carry out intelligent decision;

On-line programming and upgrade; 
The system can meet different requirements of communication with multiple communication interfaces;

The system should be equipped with standby power aim at characteristics of easy blackout during the busy time in the farm.

The design considered protecting measures of over-voltage, over-current and electrostatic, as well as the appropriate lightning protection, surge-proof ability, ensure the stability and reliability of all kinds of sensors and capacity for adapting to the harsh environment.

In view of the above system requirements, the design of seedling-nursery house controller device mainly used a method from the top down ${ }^{[2]}$, which firstly defined the overall task, then decompose into sub task. The larger, more complex and difficult problem was decomposed into a number of small, simple and easy ones to solve, and pay attention to the connection and interaction between subtasks. Some occasions also used bottom-up design method, combining with the present circuit, module and device to solve the problem quickly and efficiently. The seedling-nursery house controller has a reasonable division of labor between the software and hardware and cooperation with each other, through the software to complete the complicated calculation, system management and control, through the hardware implement a specific function. Specific analysis technique is shown in figure 1.

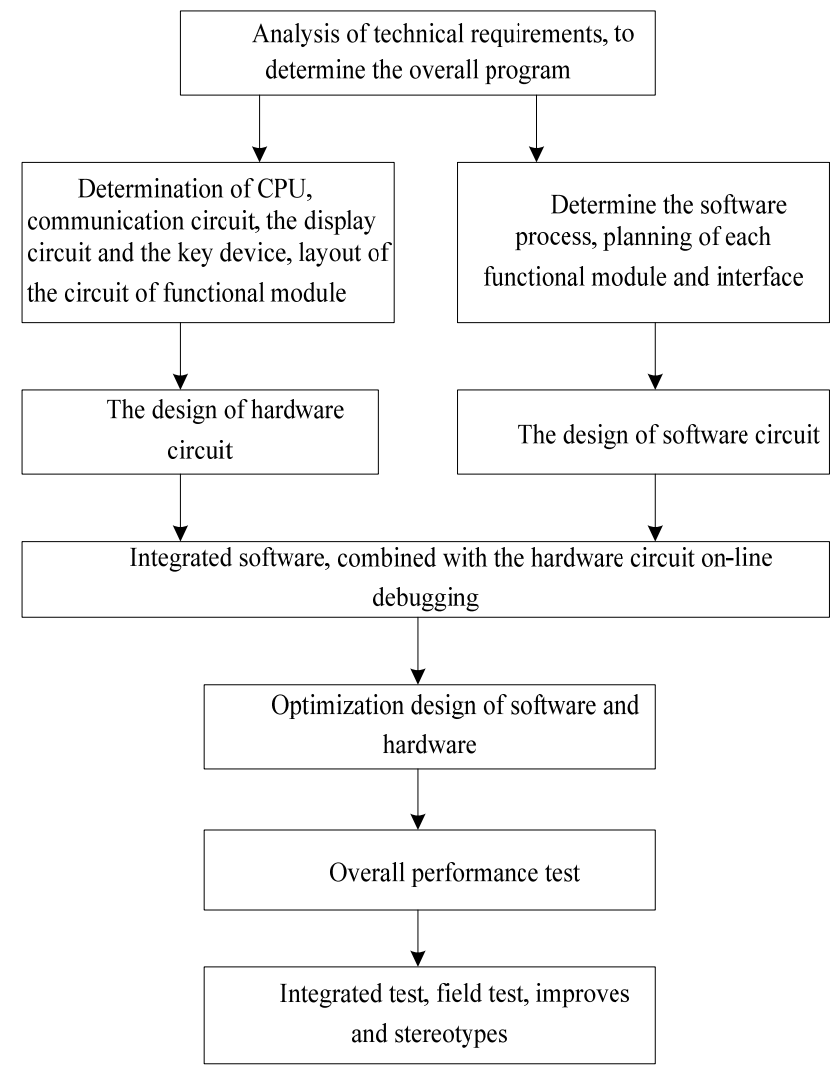

Figure 1 System technology roadmap

\section{THE DESIGN FOR HARDWARE MODULE OF THE SYSTEM AND FUNCTION DIVISION}

\section{A Micro controller selections}

The system hardware circuit structure diagram as shown in figure 2 .

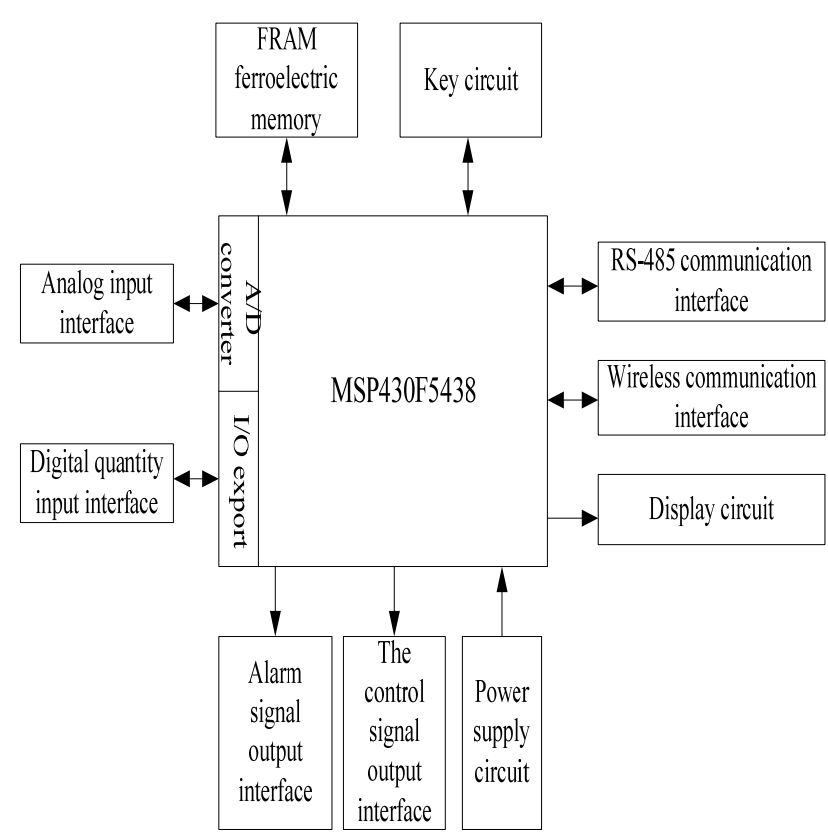

Figure 2 Block diagram of system structure

The main function of the system includes data acquisition and monitoring of the digital quantity and analog quantity, control function, function setting, time function, communication function, time recording and reporting functions, remote control function, self diagnosis function and protection of power source failure function. Micro controller uses a battery to supply power, so the selection of the CPU emphatically the low power, low voltage, high load capacity are considered, the United States of America TI company's MSP430 series of single-chip has a unique advantage in these areas, and has a plurality of USCI export, to meet the different communication mode request of the system, in addition, from price considerations, MSP430F5438 is an extremely low power Flash type, 16 bit RISC instruction set chip, has rich on-chip peripherals. The Single-chip microcomputer has the following characteristics:

Extremely low power, MSP430F5438 is a 16 bit single chip microcomputer which has the lowest power consumption in industry currently.With 5 different working mode, working current is different as the working mode differs, less than $400 \mu \mathrm{A}$, working voltage with $1.8 \sim 3.6$ V. Digital control oscillator (digital control oscillator, DCO) can be awaken from the low power mode to the active mode in less than $5 \mu \mathrm{s}$. In Standby mode, only $2.6 \mu \mathrm{A}^{[3]}$, such that, when the program is idle, can enter the Standby mode, so that equipment in extremely low power consumption standby state, waiting until the task comes, then convert to a 
normal mode of operation, greatly reducing overall power consumption of the equipment, prolonging using time.

Powerful processing ability, the models of single-chip microcomputer is 16 bit extremely low power one based on RISC architecture, has rich addressing modes ( 7 source operand, 4 destination operand), using the RISC instruction set, 27core instruction, many chip registers, efficient look-up table method, to ensure high efficiency of the programming, more interrupt sources, can realize the interrupt nesting, flexible and convenient use ${ }^{[4]}$.

Rich on-chip peripheral module, MSP430F5438 has 12 bit $\mathrm{A} / \mathrm{D}$ precision analog converter, 32 bit hardware multiplier, 2 groups of clock modules with frequencies up to $8 \mathrm{MHz}, 3$ groups of 16 bit timer, watchdog, 4 universal serial communication interface, up to 87 universal I/O pins, IIC, SPI interface; and the port P1 and P2 have interrupting capacity, each pin can be individually selected interrupt trigger mode and separately enable interrupt.

Convenient and efficient development mode, MSP430F5438 has a JTAG interface, can conveniently achieve the download and debug of program through the JTAG debugging circuit.

Based on the above advantages of MSP430F5438, this paper selected the chip as a universal intelligent control unit of a microprocessor for seedling nursery house.

\section{B Design of each function module}

\section{1) Acquisition module}

Acquisition module mainly includes analog signal and digital signal acquisition, signal acquisition mainly refers to the external sensors, analog signal for the current signal of $4 \sim 20 \mathrm{~mA}$, the system needs current-voltage conversion, after system current signal enters the series of $100 \Omega$ precision resistor, and then comes into the MSP430F5438's built-in $\mathrm{A} / \mathrm{D}$ converter for conversion, and then sent to the processor for data processing ${ }^{[5]}$. Digital signal output from the sensor is directly connected to the MCU I/O port, through the corresponding software program for data processing. In order to improve the anti-interference ability of the system, before the analog signal and digital signal come into the single chip I/O port they were isolated, analog signal isolation with CJ-SG intelligent isolator, which has the two in two out characteristics, it is intelligent, digital, programmable; full automatic compensation of environment temperature, zero and full range; high stability, ensure the accuracy for many years unchanged; power, input, output, double loop, high isolation; in line with anti electromagnetic interference standard features related to the international electrotechnical commission IEC61000.Digital signal isolation using ADI magnetic separation chip ADUM1250, the isolation chip has the advantages of small volume, low power consumption, flexible use, high integration.

\section{2) Display module}

The information collected by intelligent general in the seedling nursery house controller (multipoint air temperature and humidity, multipoint soil moisture, multi-layer soil temperature, $\mathrm{CO} 2$ concentration, soil $\mathrm{PH}$ value, light intensity, ventilation equipment and switch state of irrigation facilities, etc.), through the serial LCD module to complete display. This module has simple interface with the CPU, only take the DATA, the WR, CS 3 line interface, and much lower than parallel LCD module to save the CPU I/O port resources. In addition, low power consumption liquid crystal drive chip meets the requirements of low power consumption.

\section{3) Control module}

The system has two control modes of manual control and automatic control, Manual control in the seedling house acquisition controller using buttons to realize, automatic control need to input parameters threshold, according to different growth stages of different environmental requirements for decision making. Either way, all need seedlings house acquisition controller output control signal to start or close electromagnetic valves, shutter device. The control circuit is the core component of the isolation light coupling and electrical relay. The unit of the hardware circuit diagram as shown in figure 3 . Control signal through isolation of light coupling, output current more than $100 \mathrm{~mA}$, the current can drive the relay work, control of the electromagnetic valve open and close, adding a light coupling isolation between the controller and relay greatly reduce the relay switching of the impact of the interference caused by the controller, improving the reliability of the controller. At the same time, the coil ends of the relay connect a diode follow current, further reduce the impact of multiple switches, and improve the reliability of system.

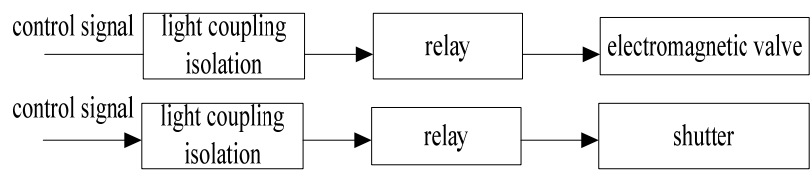

Figure 3 control module interface circuit diagram

\section{4) Communication module}

Along with the communication technology and the development of IT technique, the application of computer is becoming more and more common; in control of the seedling nursery house network communication is the inevitable trend of modern agriculture information technology development. The measured parameters of seedlings in the house control need through network into the computer, the computer monitoring and management can greatly improve the seedlings house data acquisition and intelligent control and reliability of real-time data. In the control of the communication function module, using a type of isolation RS-485 transceiver, internal integration of patent ADI magnetic coupling isolation technology, and install the protection circuit, in order to improve the communication interface of the lightning protection ability, make sure the communication interface of high reliability. The micro power launch, maximum transmission power 17 DBM $(50 \mathrm{~mW})$; Completely cover the global ISM working frequency, accord with the global ISM communication standard, need not to apply for frequency; Many channel, half-duplex wireless communication, send and receive real-time communication, the transmission distance, reliable transmission from more than 1200m; Transparent data 
transmission, automatic filtering out the air noise signal and false data (which send and receive not match), high anti-interference ability and the low bit error rate, it can provide TTL level standard UART interface, software to simulate RS232 and simulation RS485 three interface, support dormancy function, after dormancy, the whole system to minimize power consumption, +3.3 to +5.5 VDC working voltage, current $<40 \mathrm{~mA}$, emission current $<$ $100 \mathrm{~mA}$, dormancy work $<5 \mathrm{uA}$; the watchdog real-time monitoring. The SM51 each terminal functions as is shown in table 1. When SM51 and PC connected, can use a serial port RS232 level interface, when SM51 and single chip connected, can use a serial port TTL level interface ${ }^{[6]}$.

\section{5) Power supply module}

The system uses MAXIM Company DC-DC power management chips MAX857 inner power, through setting output match with the actual requirements of the voltage. When the input voltage changes, MAX857 chip provides a stable voltage value, meet requirements of CPU power, 8 40 VDC wide range of the power supply voltage, when the power supply access to switch batteries. The circuit uses protection measures such as: isolate dispose and install lightning protection, pulse groups' protection, improve the instrument anti-interference ability.

\section{6) Clock module}

In order to guarantee the collection of data corresponding with time, system uses the coprocessor DS1302 to provide time.

DS1302 is the product with a high performance, low power consumption with RAM real time clock circuit, produced by DALLAS Company, which can time year, month, day, week, hour, minute and second, has a compensation function of leap year, working voltage is 2.5 $\mathrm{V}$ to $5.5 \mathrm{~V}$, uses three line interface and the CPU for synchronous communication. The system also adopted the backup button batteries, when the system power off, DS1302 can run normally, guarantee the accuracy of the time information. At the same time, the host computer can modify clock at any time through wireless way, which ensures the whole system time synchronization ${ }^{[7]}$.

\section{7) Storage module}

In order to meet the demand that after power off data storage not lose, the system uses FM25C160 produced by the American Ramtron company to store information. FM25C160 memory has the following characteristics:

Based on a ferroelectric dielectric $16 \mathrm{~K}$ bit non-volatile memory;

Unlike other EEPROM, it can seem like RAM to bus speed operation, do not need to write delay. After sending data to equipment in SPI bus way, data is immediately written to memory array;

SPI bus speed can be as high as $20 \mathrm{MHz}$;

Has two protection mode of the hardware and software write protection;
It can be repeatedly erased 12 th power of 10 , data retention for 45 years;

The system uses SPI interface way of connection, even the system power off, the information such as the threshold value won't lost, do not need to re-write after the electricity, improve the reliability of system. At the same time, when the utility power outages, the system work in the backup power supply, every 10 minutes memory storage a gathering data. The workers can determine on the first page to display time information and period state of seedlings in nursery house, the rest pages to display environment parameters and ventilation equipment, irrigation facilities switch state.

\section{THE DESIGN OF SYSTEM SOFTWARE MODULE}

In the system software design, in order to reduce the burden of the CPU, make the CPU has more time to deal with useful operations and reduce power loss of circuit, all functions use interrupt mode. The main program deals with not much work, only some necessary initialization (including memory, clock, LCD, serial ports, A/D, etc), and open global break out into the low power consumption mode dead circulation, and wait for the interruption. Once interrupted, interrupt circulation, the CPU wake up from low power consumption mode into interrupt handling, after the break return to low power consumption dead circulation again.

\section{A The design of Data processing module}

Whether the collection of analogue or digital quantity, all need to make the necessary data processing, the system used in data processing method are: scale transform, digital filtering algorithm, linear interpolation method, the use of the algorithm improves the accuracy and reliability of data collection.

\section{B The design of CRC check module}

When the system of the data collected on the need to transmit to other machines, in order to improve the reliability of data transmission, the system designs the cyclic redundancy check code to check. The system adopts 16 bits of the CRC code, when receiving party receives, data begins to check, if not match with the check code, then send out "receiving data error" hint to the single chip microcomputer, so that the system transmit data again.

\section{The design of control unit software}

The seedling period is divided into the preparations before seeding period, seminal root growth period, first completely leaves long stretch period, deviate from milk 2 leaf period, deviate from milk 3 leaf period, transplanting preparation period. The different periods of the seedling nursery house environment the parameter requirements are also different, which in different period, higher and lower values of parameters are stored in the memory. Every time the parameters collected are compared with the limit, to determine whether action to drive electromagnetic valve or shutter device switch. When the system choose intelligent control mode, after comparison data, if overrun can instantly start or turn off the switch, such as ventilated, irrigation, etc.; If the system selection manual control mode, 
after comparison data, if overrun the sound and light alarm immediately start, and alarm messages send to users' mobile phones, to look forward to manually open or close ventilation and irrigation of the switch.

\section{System experiment and test}

The system design scheme is put into practice. After debugging software and hardware, install it in the Heilongjiang 859 farm technology research and development center of the science and technology nursery house in the testing system, in the seedling stage, seedling test running in a good condition. With the high precision sensor, it makes the data precision is very high, a large number of data greatly satisfy the demand of the farm data analysis. because of the sensor's versatility, it can be easily ported to other farms to use, just make a simple software update, the system effectively solves the shortcomings of artificial collection environment parameter, avoid major problems caused by forgetting detection and watering, which reduces manpower, material force, money; improve the process of the seedlings agricultural informatization level.

\section{CONCLUSiOnS}

In this system ,the decision of the selection of sensor and the methods of communication are closely around the house of seedling agricultural demands and the design goal of low power consumption and automatic control system, which can collect variety of environment real-time parameters, display the real-time data and automatic control, what's more, it can also be a machine to send up information to the host computer about the data collected and the switch state of electromagnetic valve, motor. It has high integration, low power consumption, strong anti-jamming performance advantages. The MSP430F538 microcontroller, use the internal $\mathrm{A} / \mathrm{D}$ converter, omit expensive cost of outside $\mathrm{A} / \mathrm{D}$ converter. Given its commonality, high reliability, etc, make it the good promotion prospects.

\section{REFERENCES}

[1] Zhang NaiMing. Facilities agriculture theory and practice [M]. Beijing: chemical industry press, 2006:1-17.

[2] Li ChangXi. Intelligent instrument principle and design [M]. Beijing: chemical industry press, 2005.

[3] Texas Instruments. MSP430F543x MSP430F541x MixedSignal Microcontroller[EB/OL]. ( 2010-01-12 ) [2011-02-15]http : //www.ti.com.cn.

[4] Shen JianHua, Yang YanQin. MSP430 16 ultra-low power single-chip microcomputer principle and practice [M].Beijing: Beijing University of Aeronautics and Astronautics, 2008.

[5] Qiao XiaoJun, Shen ZuoRui, Chen QingYun, etc. The design and implementation of agricultural facilities of environmental monitoring system [J]. Journal of agricultural engineering, 2000, (3) : 77-80.

[6] Cai WenBin, Su YiXin. Low power consumption wireless ring monitoring system design based on agricultural canopy [J]. Journal of Huazhong agricultural university, 2008, (4) : 549-552.

[7] Yu HaiYe, Ma ChengLin, Wang ZhenHua, etc. the present situation of the application of greenhouse environment control analysis in the remote control technology $[\mathrm{J}]$. Journal of agricultural machinery, $2003(6): 160-163$ 